\title{
Structure of Silver Nanoparticle Assemblies Grown at Aqueous Suspension Surfaces by Acid Vapor Diffusion
}

\author{
Seiichi Sato*, Kana Shinogi \\ Graduate School of Material Science, University of Hyogo, Kamigori-cho, Ako-gun, Hyogo, Japan
}

Received: January 20, 2017; Accepted: January 25, 2017; Published: January 27, 2017

*Corresponding author: Seiichi Sato, Assistant Professor. Graduate School of Material Science, University of Hyogo, 3-2-1 Koto, Kamigori-cho, Ako-gun, Hyogo 678-1297, Japan, Tel/Fax: +81-791-58-0161; E-mail: sato@sci.u-hyogo.ac.jp

\begin{abstract}
Silver (Ag) nanocrystals modified with mercaptosuccinic acid (MSA) were slowly assembled at air/water interfaces by dissolving vapors of hydrochloric acid $(\mathrm{HCl})$, formic acid $(\mathrm{HCOOH})$ or acetic acid $\left(\mathrm{CH}_{3} \mathrm{COOH}\right)$. By introducing $\mathrm{HCl}, \mathrm{Ag}$ nanoparticle lattices were found in the assemblies. However, the addition of $\mathrm{HCOOH}$ resulted in random aggregates of $\mathrm{Ag}$ compound crystallites, while exposure to $\mathrm{CH}_{3} \mathrm{COOH}$ induced the fusion of the Ag nanocrystals.
\end{abstract}

Keywords: self-assembly; silver nanocolloid; self-correcting process; superlattices; equilibrium growth

\section{Introduction}

Nanoparticle lattices are of considerable scientific interest since adjusting the sizes of the component nanocrystals and the surface modified molecules can tailor the electronic structures of the lattices. Recently, nanoparticle lattices have been successfully produced using spontaneous assembly processes of nanoparticles, the surface of which is modified with oleophilic or hydrophilic molecules $[1,20]$. So far, the assembly processes can be roughly categorized into two types: non-equilibrium assemblies of oleophilic nanoparticles dispersed in nonpolar suspensions and equilibrium assemblies of hydrophilic nanoparticles dispersed in aqueous suspensions. The oleophilic nanoparticles in non-polar suspensions such as toluene and hexane have been assembled by evaporating the suspensions $[3,14]$. This assembly process is usually completed in several minutes, and in such a non-equilibrium process, the quality of the resulting lattice arrangements largely depends on the initial size distribution of the nanoparticles. Conventionally, the standard deviations must be smaller than $15 \%$ of the average size to obtain quality lattices $[6,13]$.

In contrast, hydrophilic nanoparticles in aqueous suspensions can be assembled slowly (e.g. several days or several months) by adjusting the charge of the surface modifiers through $\mathrm{pH}$ control by acid. In such an equilibrium process, a narrow size distribution is not an indispensable prerequisite owing to the following self-correcting processes [18, 21]: (1) Non-uniform particles are repelled towards the boundaries of the lattice if the lattice contains component particles of various sizes, and (2) a nanoparticle trapped inside a metastable site can be released back into suspension and diffuse about the lattice surface until the most stable position is found. These two processes narrow the size distribution within the lattice, remove lattice defects, and improve lattice symmetry. To date, carboxylate-modified gold $\mathrm{Au}$ ) nanocrystals have been successfully assembled into high-quality lattice arrangements at air/water interfaces under equilibrium $[15,19]$.

In the present study, mercaptosuccinic acid (MSA) modified silver (Ag) nanocrystals were slowly assembled (i.e., assembled in equilibrium) at the air/water interface. From a viewpoint of the self-correcting nature, lattice arrangements should emerge without size fractionation of the starting nanoparticles. However, since $\mathrm{Ag}$ surface is more reactive than gold, lengthy immersion in aqueous suspensions containing acid may change the $\mathrm{Ag}$ nanocrystals into $\mathrm{Ag}$ compounds such as silver chloride (AgCl). In addition, surface modifiers may not stably protect the Ag surface, and Ag nanocrystals may be fused into larger crystals. Therefore, it is necessary to examine the reactivity of Ag nanocrystals in various acids as well as the stability of the MSA modification during self-assembly processes. Thus, herein we report structural observations of MSA-modified Ag nanocrystal assemblies grown by vapor diffusion of acids, typically hydrochloric acid ( $\mathrm{HCl})$, formic acid $(\mathrm{HCOOH})$ and acetic acid $\left(\mathrm{CH}_{3} \mathrm{COOH}\right)$.

\section{Methods}

MSA-modified Ag nanocrystals were prepared using the same method described in our previous report [22]. First, $150 \mathrm{mg}$ of MSA was dissolved in $100 \mathrm{ml}$ of methanol. Second, $1.7 \mathrm{ml}$ of a silver (I) nitrate $(5 \% \mathrm{w} / \mathrm{v})$ aqueous solution was mixed with the MSA-containing methanol. Third, $25 \mathrm{ml}$ of sodium borohydride in aqueous solution $(0.2 \mathrm{M})$ was added to the solution at a rate of $2 \mathrm{ml} / \mathrm{min}$ under vigorous stirring. All of the above preparations were performed at around $0{ }^{\circ} \mathrm{C}$.

To induce the self-assembly of the nanoparticles, $\mathrm{HCl}, \mathrm{HCOOH}$ 
or $\mathrm{CH}_{3} \mathrm{COOH}$ was dissolved by the following vapor diffusion method [23]. First, $6.0 \mathrm{mg}$ of MSA-modified Ag nanocrystals was dissolved in $3 \mathrm{ml}$ of distilled water in a glass vial $\mathrm{A}$, which was 4.0 $\mathrm{cm}$ high and had an internal diameter (ID) at the opening of $1.3 \mathrm{~cm}$. In a glass vial $\mathrm{B}$, which was $4.5 \mathrm{~cm}$ high with an ID at the opening of $1.5 \mathrm{~cm}, 5 \mathrm{ml}$ of $6 \mathrm{M} \mathrm{HCl}, 6 \mathrm{M} \mathrm{HCOOH}$ or $6 \mathrm{M} \mathrm{CH}_{3} \mathrm{COOH}$ aqueous solution was prepared. Then, the vial $\mathrm{A}$ and a set of two vials $\mathrm{B}$ were stored in a shielded vessel, which measured $10 \mathrm{~cm}$ high with an ID of $5 \mathrm{~cm}$, in a dark room. After three weeks, assemblies appeared at the air/suspension interface. The assemblies were scooped with a $\mathrm{Cu}$ grid covered with an amorphous carbon film for transmission electron microscope (TEM) observations.

Surface modification of the nanocrystals was evaluated with a Horiba FT-720 Fourier transform infrared (FTIR) spectrometer in transmission mode. TEM images and corresponding selected area electron diffraction patterns were obtained using a HITACHI H-8100 TEM operated at $200 \mathrm{kV}$ acceleration voltages. The nanocrystal size and crystallinity were evaluated using a RIGAKU RINT $2000 \mathrm{X}$-ray diffractometer with $\mathrm{Cu} \mathrm{K} \alpha$ radiation $(\lambda=1.54 \AA)$ operated at $40 \mathrm{kV}$ and $20 \mathrm{~mA}$.

\section{Results and Discussion}

Figure 1 shows the structural properties of the starting Ag nanoparticles. The FTIR result (Figure 1a) suggested the following three characteristics. First, the peak at $2550 \mathrm{~cm}^{-1}$, which corresponds to the S-H stretching mode [24], disappeared in the spectrum of the Ag nanoparticles. This indicates that the Ag nanocrystal surface is modified with MSA through the $\mathrm{S}$ atom. Second, the MSA on the nanocrystals formed carboxylate salts: The counter cations of the $\mathrm{CO}_{2}^{-}$are probably $\mathrm{Na}^{+}$because $\mathrm{NaBH}_{4}$ was used in the reduction of $\mathrm{AgNO}_{3}$. Third, the $\mathrm{Ag}-\mathrm{O}$ peak, which is usually observed as a strong peak at $540-550 \mathrm{~cm}_{-1}$ [24], was not seen. This shows that the Ag nanocrystals are successfully modified with MSA. The average diameters of the Ag nanocrystals obtained from the TEM image (Figure $1 \mathrm{~b}$ ) and X-ray diffraction (XRD) pattern (Figure 1c) were $2.8 \mathrm{~nm}$ and $2.2 \mathrm{~nm}$, respectively. The slight difference between these sizes may be due to the fusion of the nanocrystals by electron beam irradiation during TEM observation.

Figure 2 shows the TEM images of the assemblies obtained by three types of vapor diffusion. In the $\mathrm{HCl}$ vapor, lattice arrangements were grown ubiquitously. Figure 2(a) shows a typical assembly. The diffraction rings are fully explained by assuming a bulk Ag crystal structure (fcc with $a=0.409$ $\mathrm{nm}$ ). It should be noted that $\mathrm{AgCl}$ was not found, which was sometimes observed when $\mathrm{HCl}$ solution was directly mixed with the Ag nanoparticle suspensions [25]. However, the use of $\mathrm{HCOOH}$ vapor did not result in the lattice formation, but instead produced random aggregates (Figure $2 \mathrm{~b}$ ). The diffraction pattern of the aggregates cannot be explained by assuming Ag or Ag oxide crystals. Considering the chemical species contained in the suspension, the crystals are presumably Ag formates. The diffraction pattern consists of numerous rings at close distances, indicating that the assemblies are low symmetric crystals such as rhombic structures, which metal formates usually form. We will continue to evaluate the crystal structure, and the results will be published elsewhere. Our preliminary conclusion at this stage is that the addition of $\mathrm{HCOOH}$ induces the formation of $\mathrm{Ag}$ compounds, and that $\mathrm{HCOOH}$ is an inappropriate acid for producing $\mathrm{Ag}$ nanoparticle lattices. When $\mathrm{CH}_{3} \mathrm{COOH}$ vapor is introduced, Ag crystals, which ranged from 30 to $200 \mathrm{~nm}$ emerged (Figure 2c). The diffraction pattern is explained by assuming Ag twin crystals, implying that the Ag crystals were grown owing to the fusion of the Ag nanocrystals. These observations show that the stability of surface-modified Ag nanocrystals depends on interaction between surface-modifier molecules and acid ions as well as the reactivity with internal $\mathrm{Ag}$ nanocrystals with the ions.

\section{Summary}

MSA-modified Ag nanocrystals were self-assembled at aqueous suspension surfaces by dissolving $\mathrm{HCl}, \mathrm{HCOOH}$ or $\mathrm{CH}_{3} \mathrm{COOH}$ vapors into the suspensions. All three types of acid addition induced the formation of assemblies after at least three weeks, but the structures of the assemblies differed. By introducing $\mathrm{HCl}$ vapor, lattice arrangements of $\mathrm{Ag}$ nanocrystals
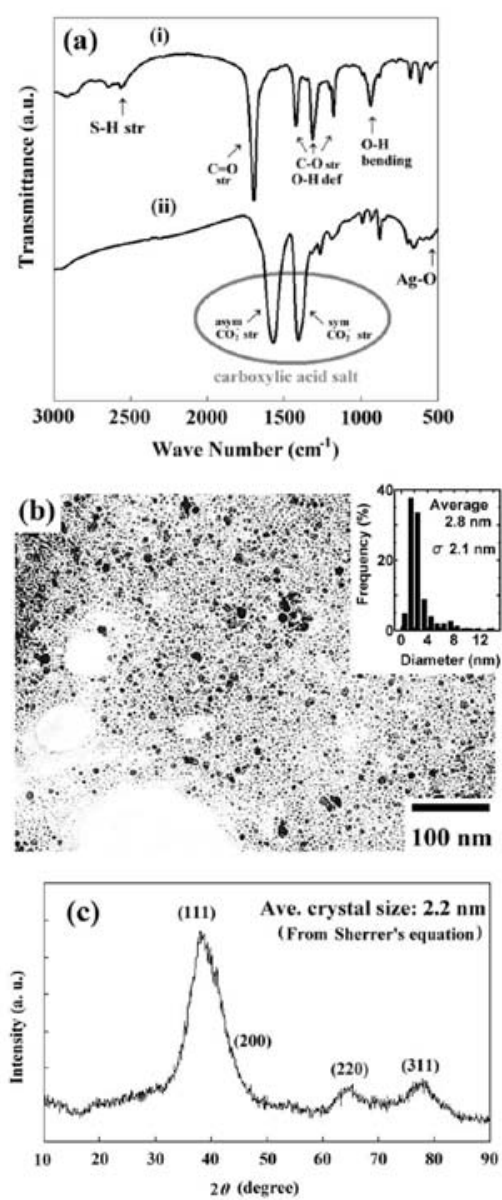

Figure 1: Structural properties of the as-prepared Ag nanoparticles: (a) FTIR spectra of (i) pure MSA and (ii) MSA-modified Ag nanocrystals, (b) TEM image and the size distribution of the Ag nanocrystals, and (c) XRD pattern. 

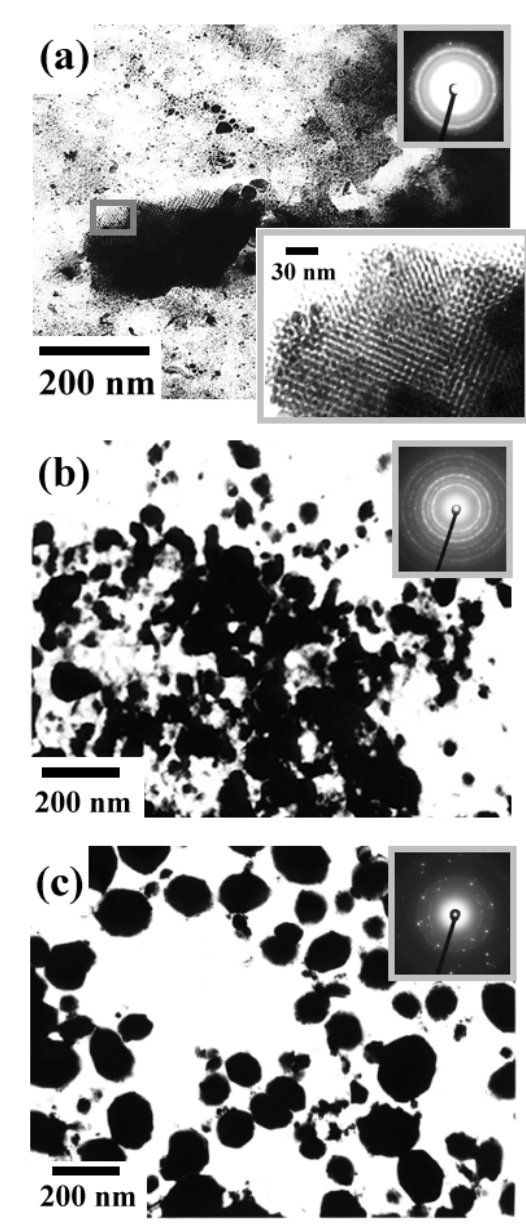

Figure 2: TEM images and diffraction patterns of the assemblies grown at suspension surfaces by exposure to vapors of (a) $\mathrm{HCl}$, (b) $\mathrm{HCOOH}$ and (c) $\mathrm{CH}_{3} \mathrm{COOH}$. In image (a), magnification of the outlined part is shown in the bottom-right corner.

were found, and no $\mathrm{AgCl}$ formation was found in the assemblies. However, exposure to $\mathrm{HCOOH}$ vapor resulted in the formation of random aggregates consisting of Ag compounds. Moreover, when $\mathrm{CH}_{3} \mathrm{COOH}$ vapor was used, the Ag nanocrystals grew 30-200 nm with twin structures, indicating that the MSA modification was unstable in the presence of $\mathrm{CH}_{3} \mathrm{COOH}$.

\section{Acknowledgements}

The authors wish to thank Professor H. Yao and Emeritus Professor K. Kimura (University of Hyogo) for their helpful discussion. This work was supported in part by a Grant-in-Aid for Scientific Research (C: 15K05994) from JSPS.

\section{References}

1. Bentzon MD, Wonterghem JV, Mørup S, Thölén A, Koch CJW. Ordered aggregates of ultrafine iron oxide particles: 'super crystals'. Phil. Mag. B. 1989;60(2):169-178. doi: 10.1080/13642818908211188.

2. Powers AS, Liao H, Raja SN, Bronstein ND, Alivisatos AP, Zheng H. Tracking nanoparticle diffusion and interaction during self-assembly in a liquid cell. Nano Lett. 2017;17(1):15-20. doi: 10.1021/acs. nanolett.6b02972.

3. Harfenist SA, Wang ZL, Alvarez MM, Vezmar I, Whetten RL. Highly oriented molecular Ag nanocrystal arrays. J. Phys. Chem. 1996;100(33):13904-13910. doi: 10.1021/jp961764x.

4. Quan Z, Xu H, Wang C, Wen X, Wang Y, Zhu J, et al. Solventmediated self-assembly of nanocube superlattices. J. Am. Chem. Soc. 2014;136(4):1352-1359. doi: 10.1021/ja408250q.

5. Brunner J, Baburin IA, Sturm S, Kvashnina K, Rossberg A, Pietsch T, et al. Self-assembled magnetite mesocrystalline films: toward structural evolution from 2D to 3D superlattices. Adv. Mater. Interfaces. 2017;4(1):1600431 [9 pages]. doi: 10.1002/admi.201600431.

6. Taleb A, Petit C, Pileni MP. Synthesis of highly monodisperse silver nanoparticles from AOT reverse micelles: a way to 2D and 3D selforganization. Chem. Mater. 1997;9(4):950-959. doi: 10.1021/ cm960513y.

7. Ji N, Chen Y, Gong P, Cao K, Peng D. Investigation on the self-assembly of gold nanoparticles into bidisperse nanoparticle superlattices. Colloids Surf. A: Physicochem. Eng. Aspects. 2015;480:11-18. doi: 10.1016/j.colsurfa.2015.03.058.

8. Sato S, Matsuda A, Sugimoto T, Kimura K. Structural and electrical characterization of three-dimensional superlattices of organic monolayer protected gold nanocrystals. Jpn. J. Appl. Phys. 2011;50: 01BH01 [3pages]. doi: 10.1143/JJAP.50.01BH01.

9. Sato $S$, Ito T, Kimura K. Refractive index regulation of gold nanocrystal superlattices by varying the nanocrystal size. Jpn. J. Appl. Phys. 2010;49: 06GJ05 [3pages]. doi: 10.1143/JJAP.49.06GJ05.

10. Chen J, Ye X, Oh SJ, Kikkawa JM, Kagan CR, Murray CB. Bistable magnetoresistance switching in exchange-coupled $\mathrm{CoFe}_{2} \mathrm{O}_{4} \mathrm{Fe}_{3} \mathrm{O}_{4}$ binary nanocrystal superlattices by self-assembly and thermal annealing. ACS Nano. 2013;7(2):1478-1486. doi: 10.1021/nn3052617.

11. Sato S, Wang S, Kimura K. Atomic alignment in particle crystals of $\mathrm{Au}$ nanoparticles grown at an air/water interface. Phys. Chem. C. 2007;111:13367-13371. doi: 10.1021/jp074126v.

12. Taleb A, Russier V, Courty A, Pileni MP. Phys. Rev. B. 1999;59:13350-13358. doi: 10.1103/PhysRevB.59.13350.

13. Murray CB, Kagan CR, Bawendi NG. Synthesis and characterization of monodisperse nanocrystals and close-packed nanocrystal assemblies. Annu. Rev. Mater. Sci. 2000;30:545-610. doi: 10.1146/annurev. matsci.30.1.545.

14. Pileni MP. Nanocrystal self-assemblies: Fabrication and collective properties. J. Phys. Chem. B. 2001;105:3358-3371. doi: 10.1021/ jp0039520.

15. Kimura K, Sato S, Yao H. Particle crystals of surface modified gold nanoparticles grown from water. Chem. Lett. 2001;30(4):372-373. doi: 10.1246/cl.2001.372.

16. Wang S, Sato S, Kimura K. Influence of organic vapors on the selfassembly of gold nanoparticles at solution-gas interfaces. Chem. Lett. 2003;32(6):520-521. doi: 10.1246/cl.2003.520.

17. Wang S, Sato S, Kimura K. Preparation of hexagonal-close-packed colloidal crystals of hydrophilic monodisperse gold nanoparticles in bulk aqueous solution. Chem. Mater. 2003;15(12);2445-2448. doi: $10.1021 / \mathrm{cm} 0217147$.

18. Sato S, Yao H, Kimura K. Equilibrium growth of three-dimensional gold nanoparticle superlattices. Physica E. 2003;17;521-522. doi: 10.1016/S1386-9477(02)00858-5.

19. Oonishi T, Sato S, Yao H, Kimura K. Three-dimensional gold nanoparticle superlattices: structures and optical absorption characteristics. J. Appl. Phys. 2007;101(11);114314 [5 pages]. doi: 10.1063/1.2743736. 
20. Yang Y, Kimura K. Highly ordered superlattices from polydisperse Ag nanoparticles: a comparative study of fractionation and selfcorrection. J. Phys. Chem. B. 2006; 110(48): 24442-24449. doi: 10.1021/jp064876p.

21. Okamoto S, Hachisu S. Ordered structure in monodisperse gold sol J. Colloid Interface Sci. 1977;62(1):172-181. doi: 10.1016/00219797(77)90079-0.

22. Chen S, Kimura K. Water soluble silver nanoparticles functionalized with thiolate. Chem. Lett. 1999; 28: 1169-1170. doi: 10.1246/ cl.1999.1169.
23. Yang Y, Liu S, Kimura K. Superlattice formation from polydisperse Ag nanoparticles by a vapor-diffusion method. Angew. Chem. Int. Ed. 2006;45(34):5662-5665. doi: 10.1002/anie.200601233.

24. Socrates G. Infrared and Raman characteristic group frequencies: table and charts, 3rd ed. New York: Wiley; 2001. pp. 210-211 and p. 324. ISBN: 0470093072.

25. Yang Y, Kimura K. Surface charge driven size evolution during the formation of self-assembled nanostructures from discrete hydrophilic silver nanoparticles. Nanotechnology. 2007;18(46):465603 [6 pages]. doi: 10.1088/0957-4484/18/46/465603. 